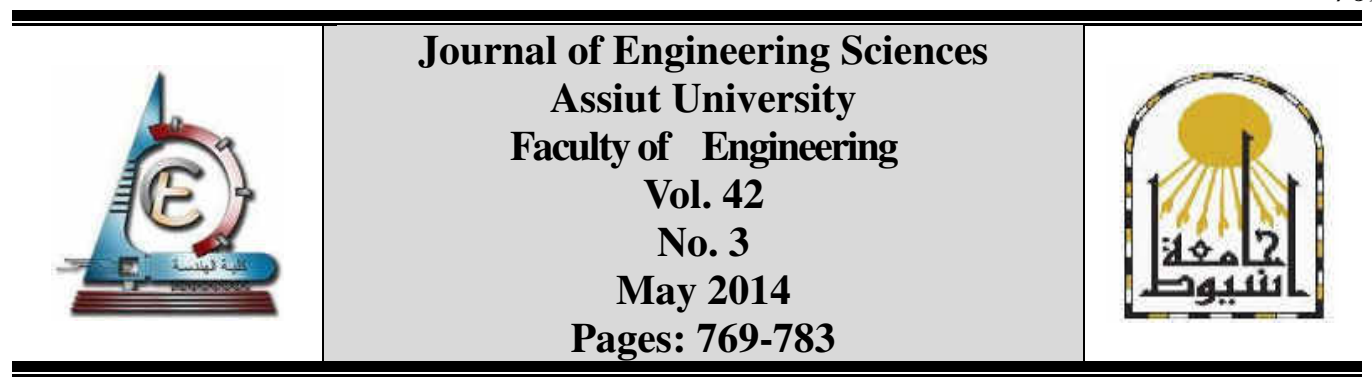

\title{
ESTIMATION OF LIGHTNING STRIKING DISTANCE TO HORIZONTAL CONDUCTOR
}

\author{
Mohamed Nayel \\ Faculty of Engineering, Electrical Engineering Dept., Assiut University, Assiut, Egypt 71518 \\ Received 1 May 2014; accepted 28 May 2014
}

\begin{abstract}
This paper attempts to investigate the horizontal conductor lightning striking distance estimation. An electromagnetic model was proposed to model a vertical downward lightning leader and an infinite grounded horizontal conductor by using the charge simulation method (CSM) to calculate the lateral striking distance. A formula between the lateral striking distance, the lightning current, and the horizontal conductor height was estimated. The proposed formula was compared with electromagnetic model and previous formulas. By the use of a similar methodology, a horizontal conductor voltage coefficient had been proposed and estimated. The proposed voltage coefficient was used to modify the electrogeometric model in order to study lightning striking rates of an unshielded horizontal conductor for different heights at either AC or DC voltages.
\end{abstract}

Keywords: Electromagnetic theory, Electro-geometric model, horizontal conductor, lightning leader, striking distance, charge simulation method, conductor voltage.

\section{Introduction}

The study of electric power reliability is one of the main concerns of electrical engineers. This issue has been researched previously by registering and assessing customer service across a considerable period of time. The analysis of such data has revealed weak points in electric power grids resulting from grid faults and lightning stokes to the electric power grid [1]. Protection and control devices were distributed in electric power grids which supplied high demand electrical power to costumers, [2]. Some areas around the equator have over 100 days of lightning strikes per year. Consequently lightning strikes to the electric power grid have become the main reason for loss of the electric power supply. In addition, where downward lightning charges travel down along the lightning leader, the electric field on the surface of conducting objects increases. Both, the direct lightning current and the induced current due to lightning discharges cause damage to equipment [3-4]. Therefore, lightning protection strategies are based on shielding electric power equipment by using shielding wires or rods. When the lightning leader bypasses the shielding systems and strokes the electric power grid, the distributed arrangements of surge arrestors protect different electric power components. However, selecting the location of surge arrestors is a complex process, as it must be conducted based on the electromagnetic 
transient of the electric power grid. Usually, the electric power grid demonstrates a dynamic behavior and varies with time [5-6]. As a result, an effective design of the lightning shielding system is a high priority in order to defend electric power grids. To achieve this objective, different techniques have been used to estimate lightning strike distance to grounded objects and the design of shielding system.

\subsection{Electro-geometric model (EGM)}

The Electro-geometric model is one of the techniques that have been successfully employed to design a shielding system and to provide a scientific basis for calculating the annual number of lightning strokes to electric power components and buildings [7-12]. As the downward leader approaches the ground, a point of the discrimination is reached for a final leader step. In contrast, other models simulate the upward directed leaders from objects [13-16]. The EGM portrays this concept with the use of striking distances [7]. This is the distance between the lightning leader tip and the conducting object, when the object surface electric field reaches a critical electric field of air breakdown and an upward leader starts from the object to meet lightning downward leader. A number of equations have been proposed for determining the striking distance and the principle examples are listed as follows [8-10]:

$$
\begin{array}{llrl}
r_{s} & =2 I+30\left(1-e^{-I / 0.68}\right) & & \text { Darveniza } \\
r_{s} & =10 I^{0.65} & & \text { IEEE, Love } \\
r_{s} & =9.4 I^{2 / 3} & & \text { Whitehead } \\
r_{s} & =8 I^{0.65} & & \text { IEEE } \\
r_{s} & =3.3 I^{0.78} & & \text { Suzuki } \\
r_{s} & =1.9 I^{0.9} & & \text { V. Cooray, et.al }
\end{array}
$$

where $r_{s}$ is the striking distance in $\mathrm{m} ; I$ is the magnitude of the lightning current in kA.

Based on the observations of the lightning strikes to different transmission lines at different conditions, Eriksson proposes a lateral striking distance equation with a dependence on conductor height $h[11]$ :

$$
D_{s}(I, h)=0.67 I^{0.74} h^{0.6}
$$

Rizk also studied the effect of conductor height on the lateral lightning striking distance [12], for a current ranging from 5 to $31 \mathrm{kA}$ and a conductor height range from 10 to $50 \mathrm{~m}$.

$$
D_{a}(I, h)=1.57 I^{0.69} h^{0.45}
$$

The Electro-geometric model (EGM) was subsequently modified to consider different parameters, such as surrounding objects, transmission line sag, and ground slope lightning slope weather conditions [17-22]. Fig. 1 shows the horizontal conductor collecting locus, as lightning strikes the horizontal conductor when reach arc bca while the remaining strikes go to ground. The attractive radius $D_{k}$ of lightning stroke with current amplitude $I_{k}$ is given by:

$$
D_{k}=D_{s v} \quad \text { for } h>r_{g}
$$




$$
D_{k}=\sqrt{D_{s v}^{2}-\left(r_{g}-h\right)^{2}} \quad \text { for } h<r_{g}
$$

Where $r_{g}$ is the lightning striking distance to ground, and $D_{s v}$ is the lightning striking distance to horizontal conductor.

If the length of the horizontal conductor is $L$, then the collection area $A_{k}$ corresponding to a lightning strike current amplitude $I_{k}$ is given by:

$$
A_{k}=2 D_{k} L
$$
by:

The number of lightning strikes to horizontal conductor of lightning current $I_{k}$ is given

$$
N_{k}=A_{k} P_{e k} N_{g}
$$

where $N_{g}$ is the ground flash density, $P_{e k}$ is the probability of lightning current $I_{k}$

Then, the annual number of lightning strokes collected by the horizontal conductor $(\mathrm{N})$ is given by:

$$
N=N_{g} \sum_{k=1}^{m} A_{k} P_{e k}
$$

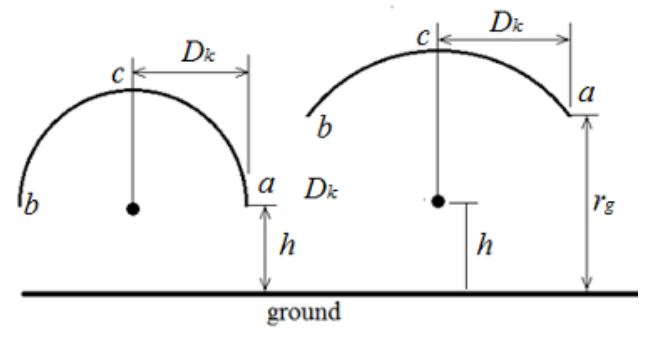
(a) $h \geq r_{g}$
(b) $h<r_{g}$

Fig. 1. The attractive locus for lightning stroke with current $\mathrm{I}_{\mathrm{k}}$.

This paper focuses on estimating the striking distance by considering the effect of lightning current, horizontal conductor height and horizontal conductor voltage in an attempt to improve the electro-geometric model. The lightning striking distances are calculated by using the electromagnetic model for different horizontal conductor heights up to $150 \mathrm{~m}$ and under different lightning currents up to $200 \mathrm{kA}$. The effect of horizontal conductor voltage on lightning striking distance has been analyzed by proposing a voltage coefficient. The proposed equation and modification for the striking distance to horizontal conductor have been used to modify the Electro-geometric model of the unshielded horizontal conductor.

\section{The electromagnetic model}

A horizontal conductor at height $h$ has been exposed to a downward lightning leader, as shown in Fig. 2. By using the charge simulation method, an electromagnetic model has been constructed to study the lightning strike to this horizontal conductor. The electromagnetic model consists of a downward lightning leader and horizontal conductor. 


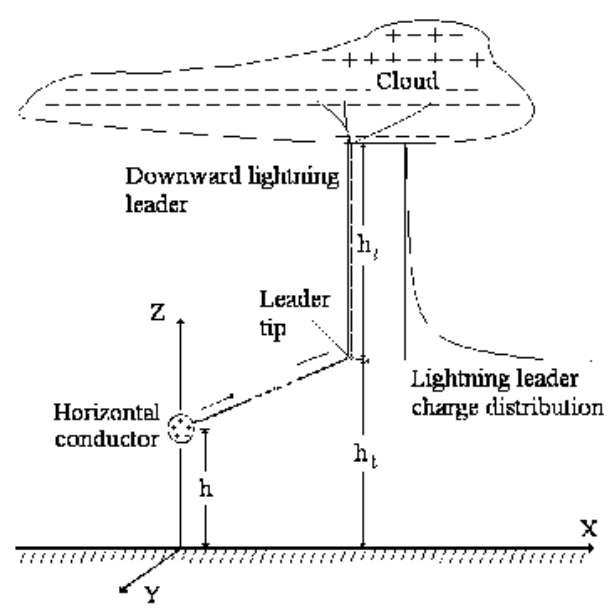

Fig. 2. Schematic arrangement of a horizontal conductor stroked by the downward lightning leader.

\subsection{Lightning leader modeling}

In this paper, the charge decaying distribution along the stepped leader was assumed to be exponentially of a negative charge as [23-24].

$$
\rho(z)=\rho_{s} e^{-\alpha\left(z-h_{s}\right)}, \alpha=\frac{\ln \left(\rho_{c} / \rho_{s}\right)}{\left(h_{c}-h_{s}\right)}
$$

where, $\rho_{s}$ is the charge density $(\mathrm{C} / \mathrm{m})$ at the tip of leader stroke $\left(z=h_{s}\right), \rho_{c}$ is the charge density $(\mathrm{C} / \mathrm{m})$ at cloud base $\left(z=h_{c}\right), \mathrm{h}_{\mathrm{c}}$ is the cloud height above ground $(\mathrm{m}), \mathrm{h}_{\mathrm{s}}$ is the height of the leader stroke tip above ground $(\mathrm{m})$.

The downward lightning leader length $h_{l}$ was assumed to be constant and equal to $3 \mathrm{~km}$ and $\rho_{c} / \rho_{s}=0.05$. This value resulted to $\alpha=10^{-3}$. The downward lightning leader was simulated by $n$ discrete line charges along the positive $Z$ direction.

The downward lightning leader was simulated by $N$ vertical discrete constant charge densities in $Z$ direction. The charge density for $n$ segment would be as follows $\left(\rho_{\mathrm{nl}}\right)$ :

$$
\rho_{n}=\frac{\rho_{s} e^{\alpha h_{s}}}{-\alpha\left|z_{n-1}-z_{n}\right|}\left(e^{-\alpha z_{n-1}}-e^{-\alpha z_{n}}\right)
$$

The potential of the downward lightning leader at any point in the space was expressed as:

$$
V=\sum_{n=1}^{N} P_{n} \rho_{n}
$$

where $P_{n}$ is the potential coefficient for $n^{\text {th }}$ vertical discrete constant charge density (shown in Appendix I).

\subsection{Horizontal conductor modeling}

The surface charges on the horizontal conductor were not uniform, due to the presence of downward lightning leader. The surface charges on horizontal conductor along the 
positive $Y$ direction was simulated by $N_{r}$ discrete line charges uniformly distributed around a factious coaxial cylinder extending along the horizontal conductor length and having a radius equal to a fraction $\beta$ of the horizontal conductor radius $r$. To account for the nonuniform distribution of the charge along the positive $Y$ direction, each of the discrete line charge was divided into $N_{a}$ sections, of which $\left(N_{a}-1\right)$ finite sections and a semi-infinite section at the end. The length of $n$ finite sections was $e^{m}\left(e^{(n+1)}-e^{n}\right)$. The starting distance of the semi-infinite line charge $d_{s}=e^{m(N a+1)}$, where $m$ is a constant and is chosen to be 1.84 [24-25].

In addition to different line charges at the radial and axial directions inside the horizontal conductor, $N_{r}$ point charges were placed at the intersection of the factious lines extension with $X-Z$ plane as shown in Fig. 2. These point charges are recommended in order to increase the boundary and check points at intersections of the horizontal conductor with $X-Z$ plane, and also, due to the presence of downward lightning leader in $X-Z$ plane [24-25]. The assumed factious charges had $N_{r} \times N_{a}+N_{r}$ unknown charges.

To maintain zero potential at the ground plane, images of the simulation charges were considered with respect to this plane. To satisfy the boundary conditions, boundary points have been chosen on the horizontal conductor surface as shown in Fig. 2. A set of equations was then formulated at a set of boundary points selectively placed on the horizontal conductor surface to determine these unknown charges. The number of boundary points is equal to the number of the unknown charges. The potential at each boundary point in the presence of downward leader was expressed as:

$$
\sum_{f l n=1}^{N_{a} N_{r}} P_{f l n} \rho_{f l n}+\sum_{s f l n=1}^{N_{a}} P_{s f l n} \rho_{s f l n}+\sum_{p n=1}^{N_{a}} P_{p n} Q_{p n}=V-V
$$

where $P_{f l n}, P_{s f l n}, P_{p n}$ are potential coefficients calculated at the boundary point due to finite line charges, the semi-infinite line charges and point charges respectively (see Appendix II), $V$ is the horizontal conductor voltage, $V$ is the voltage at the boundary point due to downward lightning leader.

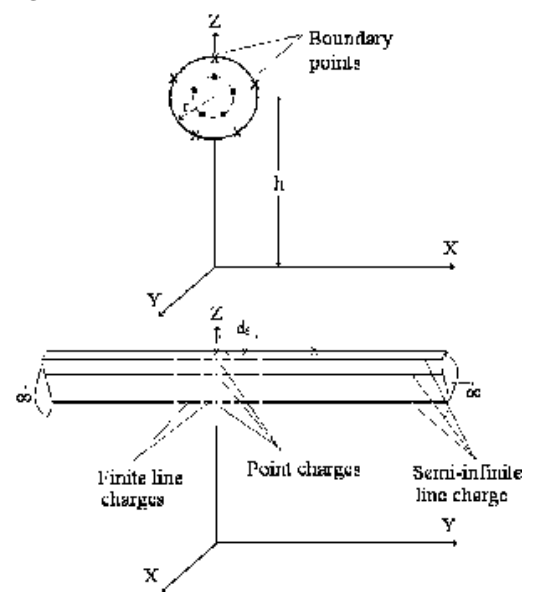

Fig. 3. Charge simulation of the horizontal conductor

The voltages at check points were calculated at different cases and compared with the conductor voltage to estimate the error. These different charges arrangements have demanded maximum error of less than $0.002 \%$. 
It is considered that lightning would strike the grounded horizontal conductor if there is an upward-directed leader from the surface of the object, as shown in Fig. 3. The following criteria were tested to estimate whether that lightning leader would strike the horizontal conductor, or not [26-28]:

- The critical electric field in Equation 18 was considered as an indication of the upward lightning leader inception. The critical ionizing electric field at the horizontal conductor surface was assumed to satisfy the requirements of Peek's law.

$$
E_{c}=3000 \delta m\left(1+\frac{0.03}{\sqrt{\delta r}}\right)
$$

where, $\delta$ is the relative air density, $r$ is the radius of the horizontal conductor; $m$ is the surface roughness factor.

- To ensure sustained progress of the upward lightning leader, the main electric field for a gap of a $10 \mathrm{~m}$ length around horizontal conductor generated by downward lightning leader, exceeded $230 \mathrm{kV} / \mathrm{m}$ [26-28]. This electric field intensity is necessary to break down this air gap.

In previous work [24], a lateral striking distance occurred when the voltage of cylindrical gap around horizontal conductor reaches the breakdown voltage of this gap. An equation of $r_{s}=a I^{b} h^{c}$, then exponential coefficient was estimated to consider voltage effect. The estimated equation was used to modify electro-geometric model to calculate shielding failure probability for high voltage AC and DC transmission lines.

\section{Electromagnetic model results}

\subsection{Horizontal conductor at zero potential}

The attractive area around 500 HVDC transmission line was studied before and shows that all shielding areas appears lateral to the transmission line [23]. Fig. 4 shows the attraction area around the horizontal conductor at a height $50 \mathrm{~m}$ due to the lightning current $31 \mathrm{kA}$. It shows that the lateral striking distance is longer than the normal striking distance. So, the lateral striking distance is estimated in this paper to be considered in EGM.

The influence of the horizontal conductor height on the lateral striking distance of the lightning leader to the grounded horizontal conductor under different lightning current is shown in Fig. 5. The horizontal conductor shows that as the lightning current increases, the lateral striking distance increases. Moreover, as shown in Fig.5, the lateral striking distance of the grounded horizontal electrode depends on its height.

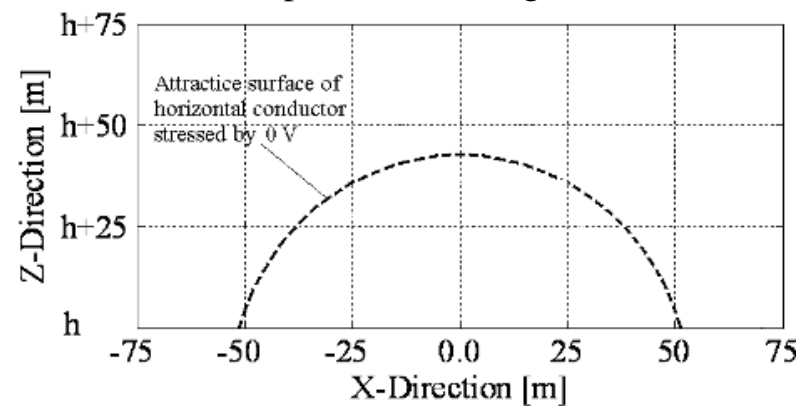

Fig. 4. Attractive surfaces of grounded horizontal conductor. 


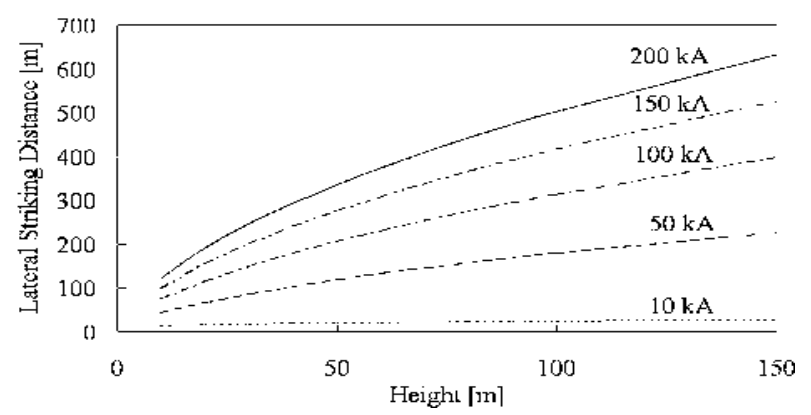

Fig. 5. Lateral striking distance vs. lightning current for grounded horizontal conductor.

The horizontal conductor height is fixed and a second order polynomial equation of lateral striking distance for each horizontal conductor height $h$ is estimated:

$$
D_{s}=A I^{2}+B I+C
$$

The coefficients $A, B$ and $C$ form a set of $n$ equations with the horizontal conductor height. A relation between the coefficients $A, B$ and $C$ and the horizontal conductor height is estimated and the following results are obtained:

$$
D_{s}(I, h)=A I^{2}+B I+C
$$

where, the coefficients $A, B$ and $C$ could be estimated by

$$
A=-0.0004 h^{0.625}, B=0.22 h^{0.625}, C=0.0
$$

The lateral striking distance obtained by the use of the electromagnetic model shows a good agreement with the lateral striking distance by using Eriksson equation (7) as shown in Fig. 6. The main deviation between the electromagnetic results and the Eriksson equation is $1.35 \mathrm{~m}$ for current range up to $200 \mathrm{kA}$ and a horizontal conductor height up to $150 \mathrm{~m}$.

Fig. 7 shows a scattering plot of the calculated lateral striking distance of grounded horizontal conductor by employing the Eriksson formula (7), Rizk formula (8), and proposed formula in Equation (19). The results show a good agreement between the proposed equation and both the formulas of Eriksson and Rizk. The main deviation between the Eriksson equation and the proposed formula in Equation (19), and between the Rizk equation in (8) and the proposed equation (19) are $7.8 \%$ and $7.4 \%$ respectively for a current range of up to $200 \mathrm{kA}$ and a horizontal conductor height up to $150 \mathrm{~m}$.

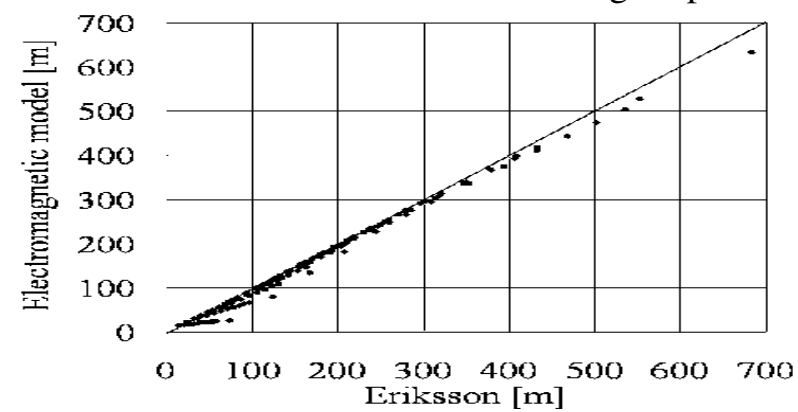

Fig. 6. The Scattering plot of the calculated lateral striking distance of grounded horizontal conductor by using different conditions compared to that by Eriksson equation (7). 


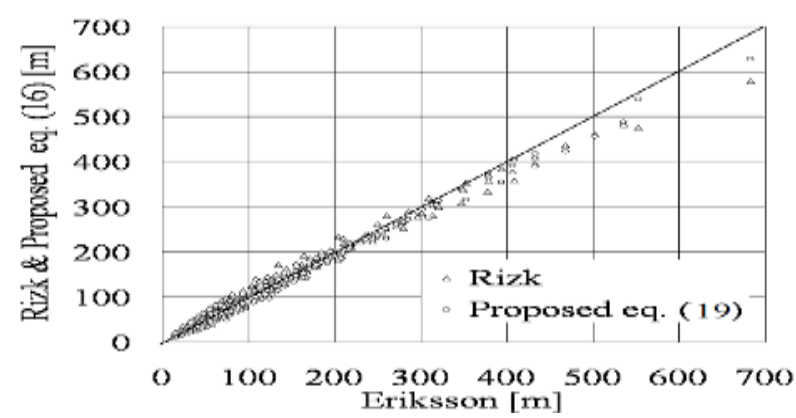

Fig. 7. The Scattering plot of the calculated lateral striking distance of grounded horizontal conductor by using Eriksson formula in (7), Rizk formula in (8) and proposed equation (19).

\subsection{A Horizontal Conductor Stressed by $+v e$ or-ve Voltage}

It was then observed that, when the ultra-high voltage (UHV) was built, more lightning accidents occurred than were originally calculated. Subsequently, it was found that, the actual measured values obtained from the lightning observations were different from the calculated values during the design stage [29].

Fig. 8 shows the lateral striking distance to a horizontal conductor at a height of $50 \mathrm{~m}$ when the horizontal conductor is stressed by DC voltage. This illustrates that the lateral striking distance increases with an increase in positive voltage of the horizontal conductor, where the lightning leader has a negative charge, and then vice versa for a negative voltage. This arises from the negative charge of the lightning leader and the positive voltage of the horizontal conductor increases in the gap potential, otherwise, the negative charge of lightning leader and the negative voltage of the horizontal conductor decrease the gap potential around the horizontal conductor.

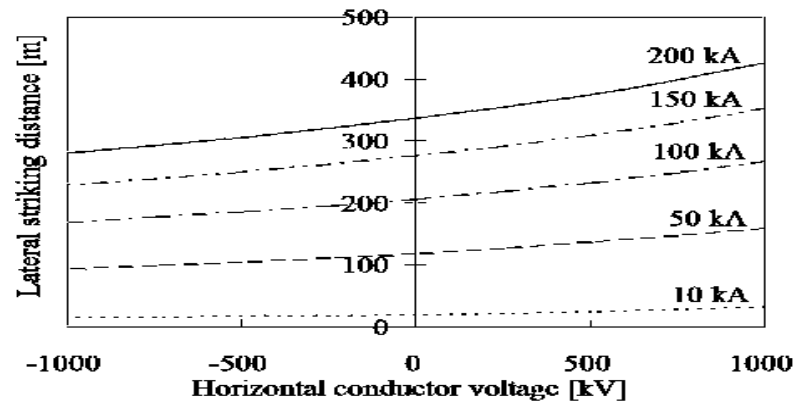

Fig. 8. The Lateral striking distance for horizontal conductor at height of $50 \mathrm{~m}$ with applied voltage varies from $-1 \mathrm{MV}$ to $+1 \mathrm{MV}$.

The lateral striking distances were obtained for a horizontal conductor at heights of 50 $\mathrm{m}$ and $20 \mathrm{~m}$, and at different horizontal conductor voltages from -1 MV to $+1 \mathrm{MV}$, and for a lightning current of up to $200 \mathrm{kA}$. By dividing the ackuired lateral striking distance curves at certain voltages by the lateral striking distance of the horizontal conductor at zero potential, it results in an approximate constant ratio $k_{v}$ at that voltage. This ratio and the horizontal conductor voltage were related as a voltage constant for each horizontal conductor voltage $k_{V}$. This coefficient is applied to the modified equation in (19) or to modified Eriksson equation in (7). 
$=0.67 I^{0.74} h^{0.6} e^{b V}$

$$
D_{s v}(I, h, V)=D_{s}(I, h) k_{v}
$$

$=\left(-0.0004 I^{2}+0.22 I\right) h^{0.625} e^{b V}$ Modified proposed eq.(19)

This provides the following coefficient: $k_{v}=e^{b V}$ and $b=0.0003$ for a negative lightning current.

Fig. 9 shows a scattering plot of the calculated lateral striking distance of a grounded horizontal conductor by using the electromagnetic model, the modified proposed equation (19) and the modified Eriksson equation. This demonstrates a positive agreement between the electromagnetic model and the proposed formulas with a voltage coefficient. Furthermore, this show the mean deviations of 0.84 and $1.15 \mathrm{~m}$ from the electromagnetic model for the modified the proposed equation (19) and the modified Eriksson equation.

\section{The probability of a shielding failure to horizontal conductor by using electro geometric model}

The probability of lightning striking a horizontal conductor stressed by AC or DC voltage under different condition were calculated to analyses some observation that were noted in relation to the use of shielding failure of UHV and EHV transmission lines by using the electro-geometric model in section I1.

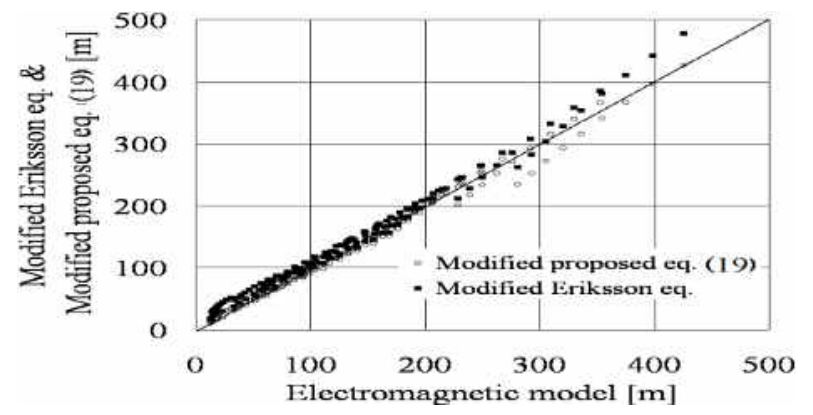

Fig. 9. The scattering plot of the calculated lateral striking distance of the horizontal conductor stressed by a voltage from -1000 to $1000 \mathrm{kV}$

The following assumptions were used in the EGM in this paper:

(1) There will be no effects of horizontal conductor till the lightning leader is within "striking distance" from the conductor.

(2) The lightning leader develops in a vertical direction.

(3) The lightning leaders experience only one strike.

(4) The lateral striking distance is a function of lightning current amplitude, horizontal conductor height and horizontal conductor voltage. In this paper the modified Eriksson equation is used to obtain the lightning striking distance to horizontal conductor.

(5) The striking distance to the ground was assumed [16] to be equal to the lightning striking distance to a horizontal conductor of equations (1) to (5). In this paper, equation (4) is used to obtain lightning striking distance to ground $r_{g}=8 I^{0.65}$.

Assuming a histogram of the amplitude of lightning currents to a flat ground is available, and that it consists of $K$ discrete current values, the $\log _{10}\left(P_{e k}\right)=0.05-I_{k} / 74$ would 
be the probability of current $I_{k}$ in China, where the ground flash density is assumed to be 0.015 [23-24]. The number of lightning strikes to different horizontal conductors and lightning stroke of a negative charge are calculated by using the electro-geometric model in section I1, and based on the previous assumptions.

Fig. 10 shows the number of lightning strikes to horizontal conductors per 100 kilometer per year. The number of lightning strikes to a grounded horizontal conductor increases as the horizontal conductor height increases. Because the lightning charge is negative, the number of lightning strikes to a horizontal conductor when it is stressed by negative voltage is less than that when the conductor is stressed with zero potential and also decreases as the conductor negative voltage increases. The opposite occurs in the case of lightning stressing the horizontal conductor with positive voltage. The lightning stroke to horizontal conductor stressed by an AC voltage is somewhat higher than that when it is stressed with zero potential and this increases as the horizontal conductor at $\mathrm{AC}$ voltage increases.

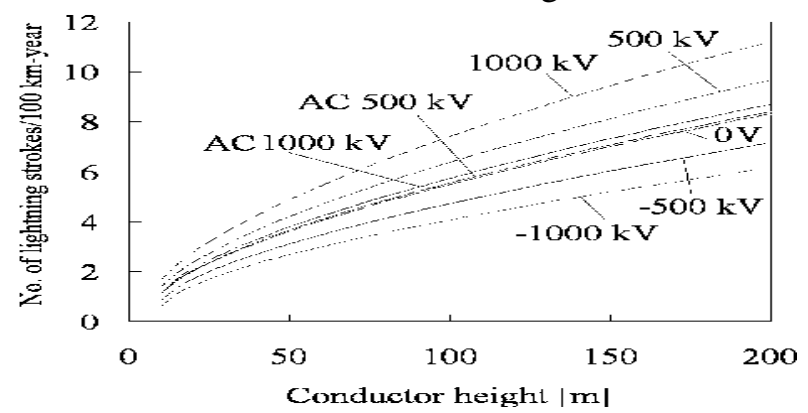

Fig. 10. The number of lightning strokes to different horizontal conductors and the lightning strike of negative charge.

\section{Conclusions}

From the results of the above calculations the following conclusions can be drawn.

- The lateral striking distance to a horizontal conductor increases as the lightning current increase, and as the horizontal conductor height increased.

- The electromagnetic model was used to obtain the lateral striking distance instead of observed results. The methodology used in estimating the lightning striking distance from electromagnetic results conducted accurate formula. As the proposed formula showed good agreement with the electromagnetic model, the Eriksson equation and the Rizk equation.

- For a negative lightning charge, the lateral lightning striking distance increases if the horizontal conductor is stressed by +ve voltage and vice versa. The methodology used to obtain the lateral striking distance was used successfully to obtain the voltage multiplied coefficient.

- The results of modification to the proposed equation and to the Erikson equations by using obtained voltage coefficient agree with the electromagnetic model results.

- The Electro-geometric model was modified successfully to consider the horizontal conductor voltage by using the voltage coefficient.

- Stressing a grounded horizontal conductor by negative voltage is attracts fewer lightning strikes than stressing the horizontal conductor by positive voltage or AC voltage. 
- The shielding system to a negative pole would be much more effective than the shielding system to the positive pole for negative lightning charges.

- The results explain the differences between the previous calculated shielding failure numbers and observed the occurrences for UHV DC/AC transmission lines.

\section{REFERENCES}

[1] R. Billinton, Z. Feng," Identification of major outage years in utility reliability performance", Electric Power Components and Systems, vol. 36, pp. 525-539, 2008.

[2] L. Silva, R. Pereira, J. Abbad, J. Mantovani," Optimized allocation of control and protective devices in electric distribution systems", Electric Power Components and Systems, vol. 38, pp. 1-21, 2010.ents in simple down conductors", Electric Power Components and Systems, vol. 40, pp. 1533-11543, 2012.

[3] V. Hegde, G. R. kunkolienker, U. Kumar," Numerical electromagnetic analysis of current and voltage distribution along down conductors and towers hit by direct lightning", Electric Power Components and Systems, vol. 38, pp. 1-21, 2011.

[4] V. Shivanand, V. Hegde, U. kumar, " On the influence of neighboring conducting objects on the induced currents in simple down conductors due to nearby lightning strike", vol. 40, pp. 1533-1543, 2012.

[5] SH. Taheri, A. Gholam, Mizaei, “ Study on the behavior of polluted insulators under lightning impulse stress”, Electric Power Components and Systems, vol. 37, pp. 1321-1333, 2009.

[6] M. Nafar, G. B. Gharehpetian, T. Niknam," A new parameter estimation algorithm for metal oxide surge arrester", Electric Power Components and Systems, vol. 39, pp. 696-712, 2011.

[7] Vernon Cooray, "On the attachment of lightning flashes to grounded structures with special attention to the comparison of SLIM with CVM and EGM", Journal of Electrostatics, vol. 7 , pp. $577-581,2013$.

[8] IEEE Std 1243-1997, "IEEE guide for improving the lightning performance of transmission lines", IEEE Inc., New York, USA, 1997.

[9] IEEE Std 1410-1997," IEEE guide for improving the lightning performance of electric power overhead distribution lines", IEEE Inc., New York, USA, 1997.

[10] V. Cooray, V. Rakov, N. Theethayi, “ the lightning striking distance-Revisited”, Journal of Electrostatics, vol. 65, pp. 296-306, 2007.

[11] A. J. Erikson," An improved electromagnetic model for transmission line shielding analysis", IEEE trans. on Power Delivery, vol. 2, pp. 871-886, Oct. 1987.

[12] F. A. M. Rizk," Modeling of transmission line exposure to direct lightning strokes", IEEE Trans. on Power Delivery, vol. 5, pp. 1983-1997, Oct. 1990.

[13] F. S. Young, J. M. Clayton, A. R. Hileman," Shielding of transmission lines", IEEE Trans. on Power Apparatus and Systems, vol. 82, pp. 132-154, 1963.

[14] Cigre Working Group 01 (Lightning) of Study Committee 33 (Overvoltages and insulation coordination), " Guide to procedures for estimating the lightning performance of transmission lines", Cigre Brochure 63, Paris, October 1991.

[15] L. Dellera, E. Garbagnati," lightning stroke simulation by means of the leader Propagation model parts I and II", IEEE trans. on Power Delivery, vol. 5, no. 4, pp. 2009-2029, October 1990.

[16] Abdul. M. Mousa, K. D. Srivastava," Modeling of Power Lines in lightning incidence calculations”, IEEE Trans. on Power Delivery, vol. 5, No. 1, pp. 303-310, January 1990.

[17] M. Nayel,“ Air conditions effects on lightning attractive distance" International Journal of Pure And Applied Science, vol. 1, no. 4, pp 50-56, April 2008.

[18] M. Nayel," Study of Air Terminals Shielding Due to Lightning Leader Slant" JES Journal of Engineering Science of assiut University, vol. 37, no. 1, January 2009. 
[19] A. M. Mousa, K. D. Srivastava," Effect of shielding by trees on the frequency of lightning strokes to power lines", IEEE Trans. on Power Delivery, vol. 3, pp. 724-732, April 1988.

[20] A. M. Mousa, K. D. Srivastava," The lightning performance of unshielded steel-structure transmission lines", IEEE Trans. on Power Delivery, vol. 4, pp. 437-445, January 1989.

[21] A. M. Mousa, K. D. Srivastava," The distribution of lightning strokes to towers and along the span of shielded and unshielded power lines", Canadian Journal of Electrical and Computer Engineering, vol. 15, pp. 115-122, 1990.

[22] C. F. Wagner," The relation between stroke current and the velocity of the return stroke", IEEE Trans. on Power Apparatus and Systems, vol. 82, pp. 609-617, October 1963.

[23] M. Nayel, Z. Jie, J. He," Analysis Shielding Failure Parameters of High Voltage Direct Current Transmission Lines" Journal of Electrostatics, vol 70, no. 6, pp.505-511, December 2012.

[24] M. Nayel " Analysis Lightning Attractive Areas around HVDC Transmission Line" Electric Power Components and Systems, vol. 37, no. 2, pp. 146-157, February 2009.

[25] M. Abdel Salam, Umar Saleh Al-Abdul-Latif, "Simulation of energized Franklin rods for lightning protection”, IEEE Trans. on Industry Applications, vol. 33, no. 3, pp. 651-659, May/June 1997.

[26] B. Wei, Z. Fu, H. Yuan," Analysis of lightning shielding failure for $500-\mathrm{kV}$ overhead transmission lines based on an improved leader progression model", IEEE transactions on Power Delivery, vol. 24, no. 3, pp. 1433-1440, July 2009.

[27] B. Vahidi, M. Yahyaabadi, M. Reza Bank Tavakoli, S. M. Ahadi," Leader progression analysis model for shielding failure computation by using the charge simulation method", IEEE transactions on Power Delivery, vol. 23, no. 23, pp. 2201-2206, October 2008.

[28] M. R. Bank Tavakoli, B. Vahidi," Shielding failure rate calculation by means of downward and upward lightning leader movement models: effect of environmental conditions", Journal of Electrostatics, vol. 68, no. 3, pp. 273-283, June 2010.

[29] J. Takami, T. Narita, S. Okabe, "Characteristics of Direct Lightning Strokes to Phase Conductors on UHV Designed Transmission Lines," IEEJ Transactions on Power and Energy, Vol.122, pp.436-441, 2002. 


\section{APPENDIXES}

\section{I- Potential coefficients for simulation downward lightning leader}

The potential coefficient $P_{n}$ of simulation finite line charge of downward lightning leader calculated at a given any point of coordinates $\left(\mathrm{x}_{\mathrm{p}}, \mathrm{y}_{\mathrm{p}}, \mathrm{z}_{\mathrm{p}}\right)$ is

$$
P_{n}=\frac{1}{4 \pi \varepsilon_{0}} \ln \left[\frac{\left(z_{n 2}-z_{p}+\gamma_{1}\right)}{\left(z_{n 1}-z_{p}+\delta_{1}\right)} \frac{\left(z_{n 1}+z_{p}+\gamma_{2}\right)}{\left(z_{n 2}+z_{p}+\delta_{2}\right)}\right]
$$

where

$$
\begin{aligned}
& \gamma_{1}=\sqrt{\left(x_{p}-x_{n}\right)^{2}+\left(y_{p}-y_{n}\right)^{2}+\left(z_{n 2}-z_{p}\right)^{2}}, \gamma_{2}=\sqrt{\left(x_{p}-x_{n}\right)^{2}+\left(y_{p}-y_{n}\right)^{2}+\left(z_{n 1}+z_{p}\right)^{2}}, \\
& \delta_{1}=\sqrt{\left(x_{p}-x_{n}\right)^{2}+\left(y_{p}-y_{n}\right)^{2}+\left(z_{n 1}-z_{p}\right)^{2}}, \delta_{2}=\sqrt{\left(x_{p}-x_{n}\right)^{2}+\left(y_{p}-y_{n}\right)^{2}+\left(z_{n 2}+z_{p}\right)^{2}}
\end{aligned}
$$

where $z_{n 1}, z_{n 2}$ are the $Z$ coordinates of the beginning and end of the nth finite simulation line charge which has a charge density $\rho_{n}, x_{n}, y_{n}$ are the $x$-and $y$-coordinates of the $n$th finite line charge.

\section{II- Potential coefficients for simulation horizontal conductor}

a) The potential coefficient $P_{p}$ of the simulation point charges of grounded horizontal conductor calculated at a given boundary point of coordinates $\left(x_{p}, y_{p}, z_{p}\right)$ is

$$
P_{p n}=\frac{1}{4 \pi \varepsilon_{0}}\left[\frac{1}{D_{p n c}}-\frac{1}{D_{p n i}}\right]
$$

where $D_{p c}$ is the distance between the point charge and the boundary point and $D_{p i}$ is the distance between image of the point charge and the boundary point. $\varepsilon_{0}$ is the permittivity of free space.

b) the potential coefficient $P_{f l}$ of simulation finite line charge of grounded horizontal conductor calculated at a given boundary point of coordinates $\left(\mathrm{x}_{\mathrm{p}}, \mathrm{y}_{\mathrm{p}}, \mathrm{z}_{\mathrm{p}}\right)$ is

$$
P_{f l n}=\frac{1}{4 \pi \varepsilon_{0}} \ln \left[\frac{\left(y_{f l n 2}-y_{p}+\gamma_{1}\right)}{\left(y_{f l n 1}-y_{p}+\delta_{1}\right)} \frac{\left(y_{f l n 1}+y_{p}+\gamma_{2}\right)}{\left(y_{f l n 2}+y_{p}+\delta_{2}\right)}\right]\left[\frac{\left(y_{f l n 1}-y_{p}+\gamma_{3}\right)\left(y_{f l n 1}+y_{p}+\gamma_{4}\right)}{\left(y_{f l n 2}-y_{p}+\delta_{3}\right)} \frac{\left(y_{f l n 2}+y_{p}+\delta_{4}\right)}{(I)}\right]
$$

where

$$
\begin{aligned}
& \gamma_{1}=\sqrt{\left(x_{p}-x_{f l n}\right)^{2}+\left(y_{f l n 2}-y_{p}\right)^{2}+\left(z_{p}-z_{f l n}\right)^{2}}, \gamma_{2}=\sqrt{\left(x_{p}-x_{f l n}\right)^{2}+\left(y_{f l n 1}+y_{p}\right)^{2}+\left(z_{p}-z_{f l n}\right)^{2}}, \\
& \delta_{1}=\sqrt{\left(x_{p}-x_{f l n}\right)^{2}+\left(y_{f l n 1}-y_{p}\right)^{2}+\left(z_{p}-z_{f l n}\right)^{2}}, \delta_{2}=\sqrt{\left(x_{p}-x_{f l n}\right)^{2}+\left(y_{f l n 2}+y_{p}\right)^{2}+\left(z_{p}-z_{f l n}\right)^{2}}, \\
& \gamma_{3}=\sqrt{\left(x_{p}-x_{f l n}\right)^{2}+\left(y_{f l n 2}-y_{p}\right)^{2}+\left(z_{p}+z_{f l n}\right)^{2}}, \gamma_{3}=\sqrt{\left(x_{p}-x_{f l n}\right)^{2}+\left(y_{f l n 1}+y_{p}\right)^{2}+\left(z_{p}+z_{f l n}\right)^{2}}, \\
& \delta_{3}=\sqrt{\left(x_{p}-x_{f l n}\right)^{2}+\left(y_{f l n 1}-y_{p}\right)^{2}+\left(z_{p}+z_{f l n}\right)^{2}}, \delta_{4}=\sqrt{\left(x_{p}-x_{f l n}\right)^{2}+\left(y_{f l n 2}+y_{p}\right)^{2}+\left(z_{p}+z_{f l n}\right)^{2}}
\end{aligned}
$$


where $y_{f l n 1}, y_{f l n 2}$ are the y coordinates of the beginning and end of the nth finite simulation line charge which has a charge density $\rho_{f l n}, x_{f l n}, z_{f l n}$ are the $X$ - and $Z$ - coordinates of the $\mathrm{n}^{\text {th }}$ finite line charge.

c) The potential coefficient $P_{s f l}$ of simulation semi finite line charge of grounded horizontal conductor calculated at a given boundary point of coordinates $\left(x_{p}, y_{p}, z_{p}\right)$ is

$$
P_{s f l n}=\frac{1}{4 \pi \varepsilon_{0}} \ln \left[\frac{\left(y_{s f l n}+y_{p}+\alpha_{1}\right)}{\left(y_{s f l n}-y_{p}+\beta_{1}\right)} \frac{\left(y_{s f l n}+y_{p}+\alpha_{2}\right)}{\left(y_{s f l n}-y_{p}+\beta_{2}\right)}\right]
$$

where

$$
\begin{aligned}
& \alpha_{1}=\sqrt{\left(x_{p}-x_{s f l n}\right)^{2}+\left(y_{s f l n}+y_{p}\right)^{2}+\left(z_{p}-z_{s f l n}\right)^{2}}, \beta_{1}=\sqrt{\left(x_{p}-x_{s f l n}\right)^{2}+\left(y_{s f l n}-y_{p}\right)^{2}+\left(z_{p}-z_{s f l n}\right)^{2}}, \\
& \alpha_{2}=\sqrt{\left(x_{p}-x_{s f l n}\right)^{2}+\left(y_{s f l n}+y_{p}\right)^{2}+\left(z_{p}+z_{s f l n}\right)^{2}}, \beta_{2}=\sqrt{\left(x_{p}-x_{s f l n}\right)^{2}+\left(y_{s f l n}-y_{p}\right)^{2}+\left(z_{p}+z_{s f l n}\right)^{2}}
\end{aligned}
$$

where $x_{s f l n}, y_{s f l n}, z_{s f l n}$ are the $x$-, $y$-, and $z$ - coordinates of the starting point of the nth semi finite simulation line charge which has a charge density $\rho_{s f l}$. 


\section{تقدير بعد ضرب صاعقة لموصل أفقي}

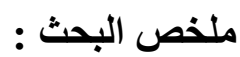

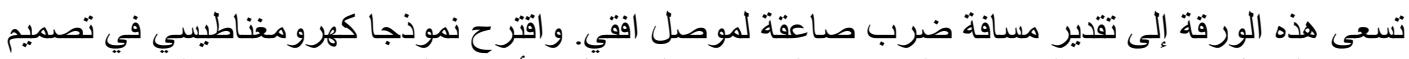

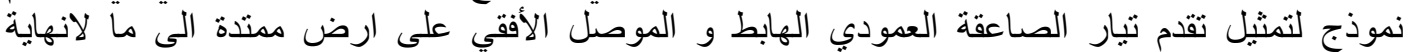

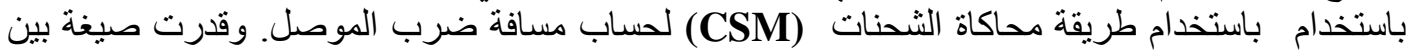

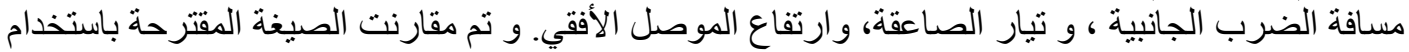

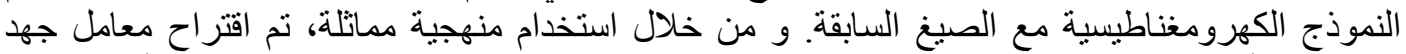

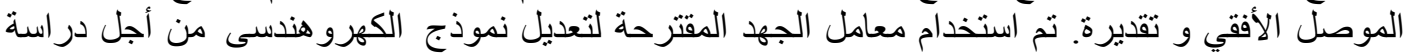

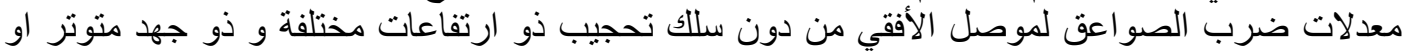

